\title{
Las Políticas culturales de proximidad en el paradigma de la ciudad creativa: el caso del programa de centros cívicos en la ciudad de Barcelona
}

\author{
María Victoria SÁnchez Belando \\ Universidad de Barcelona \\ victoriasanchez@ub.edu
}

Recibido: 29-05-2014

Aceptado: 18-02-2015

\section{Resumen}

Desde fines de los años ' 80 las ciudades experimentan un giro "creativista". El concepto de creatividad, aparece como una fuerza configuradora de las políticas económicas, sociales, culturales y urbanísticas en el contexto del post-fordismo. En Barcelona, el paradigma de la ciudad creativa influyó profundamente al denominado "Modelo Barcelona". La difusión del paradigma en el contexto europeo y su relación con el giro neoliberal de las ciudades desde los años 80 ha sido extensamente analizada, sin embargo el impacto de esta orientación en las políticas culturales de proximidad, consideradas como el resultado de la interacción entre gobierno local y actores sociales, es un terreno por explorar. El objetivo de este artículo es mostrar, en el caso de la ciudad de Barcelona, las contradicciones y déficits generados por el impacto del paradigma de la creatividad y del giro emprendedor de la ciudad en las políticas culturales de proximidad, considerando la dimensión participativa y los objetivos de profundización democrática atribuidos a estas políticas en su genesis. Para esto, examinamos la genesis, evolución y cambios de una política emblemática de la agenda socialdemócrata -el Programa de Centros Cívicos-, inscribiendo el análisis en el modelo de ciudad, el proceso de descentralización municipal y el papel de actores sociales clave como las asociaciones de vecinos durante estos 35 años de democracia.

Palabras clave: Proximidad, descentralización municipal, participación, creatividad, neoliberalismo. 


\title{
Proximity Cultural Policies in the Paradigm of the Creative City: the Case of Civic Centers Program in Barcelona City
}

\begin{abstract}
From the late ' 80 cities experienced a "creative" turn. The concept of creativity, appears as a shaping force to the economic, social, cultural and urban planning policies in the postfordist context. In Barcelona, the so-called " Barcelona Model” has been deeply influenced by the creative city paradigm. The paradigm replication in the European context and its relationship to the neoliberal turn of cities since the ' 80 s has been extensively analyzed, however, the impact of this trend on the cultural proximity policies, considered as the result of the interaction between local government and social actors, is a field to explore. The aim of this paper is to show, in the case of Barcelona city, the deficits and contradictions generated by the influence of the creative and entrepreneurial turn of the city in the cultural proximity policies implementation, considering the problem in relation to the participatory dimension and the democratic deepening objectives attributed to these policies in its genesis context. To reach this aim, we examine the socio-genesis, evolution and changes of a flagship policy for the social democratic agenda - the Civic Centers Program-, embedding its analysis in the city model, the municipal decentralization process and the role of key social actors such as neighbors associations during these 35 years of democracy.
\end{abstract}

Keywords: Proximity; Municipal Decentralization; Participation; Creativity; Neoliberalism.

\section{Referencia normalizada}

Sánchez Belando, M. V. (2015): "Las Políticas culturales de proximidad en el paradigma de la ciudad creativa: el caso del programa de centros cívicos en la ciudad de Barcelona", Política y Sociedad, 52 (1), pp. 125-152.

Sumario: 1. Introducción. 2. Institucionalización de la participación ciudadana en el modelo de descentralización barcelonés. 3. La participación social en el ámbito cultural. 4. Entre la demanda vecinal de Ateneos Populares y la respuesta del gobierno local, los Centros Cívicos. 5. Los Centros Cívicos en el en el giro emprendedor y creativista de la ciudad. 6. Conclusiones.

7. Bibliografía. 


\section{Introducción}

Desde fines de los años ' 80 asistimos a lo que podría denominarse un giro "creativista" de las políticas urbanas. El concepto de creatividad aparece como onda expansiva y fuerza configuradora de las políticas económicas, sociales, culturales y urbanísticas tras el estallido del modelo de acumulación industrial y los cambios que éste implicó en las ciudades.

En el contexto post-fordista ${ }^{2}$, los gobiernos de las antiguas ciudades industriales de Europa occidental, como Glasgow, Montpellier y Barcelona, entre otras, identificaron la cultura y la creatividad como elementos constitutivos del nuevo paradigma económico, algo que reorganizó, sustantivamente, los objetivos de la política cultural y su papel en las estrategias económicas y transformaciones urbanas (Bianchini, 1993; García, 2004a; González, 2011)

En este nuevo escenario el paradigma de las ciudades creativas (Landry y Bianchini, 1995; Florida et al., 2009) se impone con fuerza en la agenda de los gobiernos locales. La ciudad de Barcelona no ha quedado ajena al influjo de las recomendaciones de los gurús de la creatividad, progresivamente incorporadas al denominado "Modelo Barcelona".

En el caso de Barcelona, estos cambios se hacen visibles con cierto retraso respecto de las ciudades británicas y en general del entorno europeo. El triunfo de la socialdemocracia en las primeras elecciones locales en 1979 y el imperativo de afrontar el profundo déficit que legaba la dictadura en cuanto a políticas redistributivas y de bienestar (Brugué y Gomà, 1998; Morató, 2005; Borja, 2010) marcaron -aunque por poco tiempo- otro rumbo en la agenda política. Es a finales de los ` 80 y principios de los ' 90 , coincidiendo con el contexto olímpico cuando se evidencia el giro de la ciudad hacia un modelo emprendedor (Harvey, 1989; Jessop y Sum, 2000). En esos mismos años, los críticos del giro emprendedor y la instrumentalización de la cultura en las ciudades (Harvey, 1989; Zukin, 1992; Zukin, 1995; Peck, 2005; Harvey, 2001) ya empezaban a alertar sobre las contradicciones de esta tendencia.

Las críticas han apuntado a la creciente instrumentalización económica y social de la cultura y de sus productores como un fenómeno ligado al neoliberalismo: el papel de la cultura en estrategias de regeneración urbana orientadas al branding y los intereses del mercado inmobiliario (Zukin, 1989; Balibrea, 2004; Borja, 2010; Bianchini, 1993;

1 Usamos el término creativista, para enfatizar la fuerza con la que se introdujo la creatividad como un instrumento para el impulso de diversos objetivos de las políticas urbanas en el discurso de los policy makers.

2 Tomamos el término post-fordismo para referirnos al cambio de paradigma tecno-económico orientado al conocimiento que ha implicado una forma de producción flexible basada en el uso de máquinas y sistemas flexibles para garantizar economías de alcance y/o de redes, conscientes del debate en torno a si post-fordismo expresa el mejor modo de describir este cambio. (Jessop y Monedero, 2008) 
García, 2004a; Harvey, 2001) y a la elaboración de un discurso sobredimensionado sobre la capacidad de la cultura para afrontar problemas de orden social (Belfiore, 2002; Connolly, 2011).

La problemática a la que hacemos referencia se resumiría en la tensión provocada por el debilitamiento de las políticas dirigidas al bienestar de quienes habitan las ciudades y el fortalecimiento de las políticas de posicionamiento internacional de las ciudades en un mercado global altamente competitivo. Esto, en un escenario de regresión de los mecanismos de redistribución estatal y de avance de una lógica de mercado como principio configurador de la vida social, económica y política de las ciudades (Harvey, 1989; Harvey, 2013).

En el gobierno de las ciudades, estas transformaciones implicaron un nuevo pacto entre sector público y privado que cristaliza en la introducción de fórmulas de governance y de una visión estratégica en la gestión y provisión de servicios públicos. Los cambios fueron validados a partir de un nuevo discurso sobre la ciudad y sobre la relación entre sociedad civil y gobierno local, en el que la participación y la cultura aparecen como componentes centrales. En la nueva estrategia emprendedora, el énfasis en la participación se instala como un elemento desproblematizador de las tensiones entre governance y democracia (Swyngedouw, 2005; Blakeley, 2005; Geissel, 2009), algo que obliga a examinar críticamente esta relación.

Considerada esta metamorfosis del nivel local, el objetivo de este paper es responder a los interrogantes: ¿Cuál es el impacto del paradigma emprendedor de base cultural por el que ha optado la ciudad de Barcelona sobre las políticas culturales de proximidad? y ¿Cómo afecta este impacto en la dimensión participativa y de profundización democrática atribuida a estas políticas? Para responder a estas preguntas, estudiaremos la formulación, implementación y cambios del programa de Centros Cívicos en relación con el modelo de ciudad y el papel que la cultura representa en este marco, desde una perspectiva histórica que abarca estos 35 años de democracia.

Elegimos los Centros Cívicos como caso de estudio, porque se han situado en el discurso del gobierno local como una insignia de la agenda socialdemócrata para el ámbito socio-cultural, enfatizando su carácter participativo, permeabilidad y apertura hacia el vecindario en lo que respecta a programación, gestión y usos sociales. Dado este carácter en lo conceptual y la deriva de los Centros Cívicos en el contexto de las grandes transformaciones de la ciudad en las últimas tres décadas, consideramos que son un caso privilegiado de análisis a través del que es posible "tomar el pulso" del rol y las relaciones entre la administración local y el movimiento de vecinos en el diseño de una política característica del municipalismo de bienestar de los primeros años de la democracia local (Brugué y Gomà, 1998).

En este artículo defendemos que los déficits del programa de centros cívicos en términos de participación se deben principalmente a los siguientes factores: (1) El diseño de un modelo de descentralización y de participación ciudadana modelado por el giro 
emprendedor asumido por la ciudad dado en los 80 (2) Dentro de este giro, la apuesta del Ayuntamiento por actuaciones basadas en una concepción instrumental de la política cultural adecuada al contexto de la nueva economía de servicios de base cultural en detrimento de actuaciones orientadas por los principios de proximidad y democracia cultural, en un marco del declive de las políticas de bienestar en el nivel local.

El estudio de la temática aquí planteada se ha abordado de manera escasa e indirecta, así encontramos: análisis sobre el papel del tercer sector en la gestión de la cultura (Subirats et al., 2008; Lopez de Aguileta, 2000); aquellos que estudian el papel de los movimientos sociales urbanos en relación a la cultura en el paradigma de la creatividad (Novy y Colomb, 2013; Kagan y Hahn, 2011; Martí-Costa y Miquel, 2011); los que analizan el papel de la política cultural en las transformaciones urbanas en el postfordismo y su impacto en la vida social y política de las ciudades (Bianchini, 1993; García, 2004a; Degen y García, 2012) y los que focalizan en los modelos de gestión de programas y equipamientos de proximidad (Miralles, 2000; Baltà, 2007; Sánchez Belando, 2010). El estudio académico de las políticas culturales de proximidad y su dimensión participativa es un terreno aún inexplorado, en este sentido, es que consideramos la necesidad de analizarlo.

Desde el punto de vista metodológico, el artículo se basa en la realización y análisis de entrevistas en profundidad a una muestra ${ }^{3}$ significativa de cargos técnicos del Ayuntamiento, Directores de Centros Cívicos y miembros de organizaciones sociales. Además, hemos recurrido a datos de la investigación. El Sistema de la política cultural en España (CECUPS- 2008- 2011); de manera complementaria, analizamos fuentes documentales como Memorias, Presupuestos, Normativas y otros documentos elaborados por el Ayuntamiento, los Distritos y los Centros Cívicos.

\section{Institucionalización de la participación en el modelo de descentralización barcelonés}

La participación institucional, se plantea a inicios de la democracia local como un asunto prioritario de la agenda política socialdemócrata. Sin embargo, su institucionalización no puede atribuirse sólo a la acción del gobierno, ya que el movimiento de vecinos fue un actor central en este proceso, así como en la creación de servicios y

${ }^{3}$ Se realizaron un total de 22 entrevistas entre 2010 y 2014 a una muestra razonada de los principales responsables técnicos (cargos altos y medios de la administración pública, sus agencias o instituciones) de la administración local (8). También se ha entrevistado a responsables de Centros Cívicos (4), miembros de la Federación de Asociaciones de Vecinos de Barcelona (2), Miembros de organizaciones socio-culturales (6) y personas que participaron en las reivindicaciones del movimiento vecinal en distintos momentos desde 1979 hasta la actualidad (4). 
equipamientos cívicos y culturales (Andreu y FAVB, 2010) a través de una sostenida lucha iniciada a fines de la década del 60 (Bier, 1979; Borja, 1977).

Durante los años 70, Madrid y Barcelona - donde, en un contexto de creciente migración del campo a la ciudad, la periferia urbana evidenciaba la indiferencia del franquismo respecto de políticas para mejorar las condiciones de vida de las nuevas barriadas- son el principal escenario del paso de la autoorganización vecinal para la provisión de servicios básicos a la formación de un movimiento cuyo crecimiento y politización se explica en gran medida por la estratégica simbiosis entre movimiento de vecinos, partidos políticos en la clandestinidad, agrupaciones y otros colectivos antifranquistas, en un contexto fuertemente represivo (Castells, 1986: 299-301). Así las Asociaciones Populares de Vecinos ${ }^{4}$ se convirtieron en un foco de atracción y altavoz de una diversidad de sectores opuestos al régimen.

En esta espiral de ascenso reivindicativo, ya durante la democracia local, el Movimiento Vecinal formula una nueva demanda: la participación en el gobierno a través de mecanismos ad-hoc (Castells, 1986), pasando a una fase de reivindicación más bien cualitativa centrada en el debate sobre qué tipo de sistemas de participación se integrarían al Estado (Domingo i Clota y Bonet i Casas, 1998; Montero et al., 2006).

Así, se realizan actuaciones que responden en gran medida a la presión vecinal. En 1985 comienza a implementarse la descentralización del gobierno local y se definen las primeras Normas de Participación ciudadana (1986, actualizadas en 2002). Ambos procesos enmarcados en la Ley Reguladora de las Bases del Régimen Local (Ley 7/1985, de 2 de abril) y en el caso de Barcelona, en la Nueva Carta Municipal ${ }^{5}$, aunque aprobada varios años después (1999 y modificada en 2005), jugaba un papel significativo el impulso descentralizador, la participación y las formas de cooperación público-privada. Dentro del territorio español, Barcelona será pionera en estas transformaciones, que en el caso de Madrid se retrasan hasta 1988 y 1992 respectivamente (Bonet-Martí, 2012).

Entre 1979 y 1984, durante el diseño del modelo descentralizador, la discusión ${ }^{6}$ se focalizó en definir cuál sería el alcance y mecanismos de participación ciudadana en

${ }^{4}$ Hay que distinguir entre Asociaciones de Vecinos Populares, creadas por los residentes del barrio, de las Asociaciones de Vecinos Oficiales, creadas por los gobiernos municipales del Régimen o sus defensores como estrategia de control de la movilización social (Bier, 1979: 171). Según esta distinción, aquí nos ocupamos de las Asociaciones Populares a las que entendemos como parte de los denominados Movimiento Sociales Urbanos (Fainstein, S.S. y Hirst, 1995) en un sentido más amplio que el que plantea Castells (Castells y de Solís, 1985).

${ }^{5}$ Cfr. Carta Municipal de Barcelona, Título IV Participació ciutadana i drets dels veïns, Capítulo I y II, Principios generales e Institutos de Participación ciudadana. El Título VI, Capítulo I, define las condiciones de la Consorciarización y Convenios para las estructuras de governance público-privadas, cuyo rasgo central es el carácter no lucrativo de la parte privada.

${ }^{6}$ Se crearon dos espacios para canalizar el debate y generar consenso sobre la descentralización: La Comisión Ciudadana (1983) compuesta por representantes de las administraciones no municipales, enti- 
este modelo. Para el Movimiento Vecinal, era fundamental que ésta tuviera carácter administrativo y también político; es decir, que contara con herramientas eficaces para incidir en las decisiones sobre la política de los futuros diez Distritos barceloneses. En aquel momento estas transformaciones representaban un revés frente al orden político de la dictadura, caracterizado por una fuerte acumulación de poder en el Estado Central.

Sin embargo, en el nivel normativo, la descentralización tuvo finalmente un carácter dual77; (Amorós, 1999:34): como herramienta política de democratización y como instrumento administrativo para la provisión de servicios, esquema en el que las asociaciones eran vistas como un valioso recurso. Esta dualidad de la estrategia descentralizadora marcó ciertas tensiones entre las expectativas del movimiento vecinal y la necesidad del gobierno local de que la participación se produjese en la dimensión administrativa frente a los retos que imponía el contexto de recesión económica y recorte del gasto público (Blakeley, 2005).

En 1985, Jordi Borja elabora un informe de antecedentes de procesos de descentralización en el contexto europeo, en el que identifica y plantea cuestiones clave para el diseño del modelo barcelonés. En aquel documento, Borja plantea la estrategia descentralizadora como parte de un cambio político-administrativo y económico, en el marco de las respuestas de los gobiernos locales frente a la crisis europea del Welfare State de base manufacturera. De manera más particular, señala que en el escenario de crisis económica y crecimiento urbano que experimentan países como Francia o España: "los entes locales y descentralizados deberán actuar con criterios de empresa privada y, en muchos casos, sometidos a las reglas del mercado" (Borja, 1985:35).

La descentralización se inscribe, en el caso de Barcelona como una herramienta de reestructuración estatal y económica, no exenta de tensiones dadas por la difícil convivencia de interés público y privado; tensiones que luego han sido identificadas por el propio Borja como el germen de la crisis del modelo de ciudad (Borja, 2010) y que en el caso de la política cultural han sido identificadas como contradicciones inherentes a las estrategias de crecimiento económico de base cultural frente a la caída del fordismo en diversas ciudades Europeas (Bianchini, 1993; García, 2004a; Connolly, 2011).

Dentro de este paradigma de descentralización orientado al management (Amoros, 1996) el modelo de participación planteado, en la práctica, se tradujo en la cooptación de las asociaciones vecinales para la provisión de servicios y no en la creación de espacios efectivos para la deliberación y toma de decisiones. La reestructuración estatal,

dades ciudadanas (entre éstas la FAVB) y expertos y la Ponencia de la División Territorial de Barcelona compuesta por todas las fuerzas políticas presentes en el Pleno del Ayuntamiento (PSUC representado por Jordi Borja, PSC, CIU y AP) (Amorós, 1999)

${ }^{7}$ Amorós distingue entre una dimensión política de la descentralización, que contemplaría la participación en la toma de decisiones y otra administrativa, donde la participación se limita a la provisión de servicios por parte de organizaciones sociales. Esta última forma es la que Amorós identifica como predominante en la arquitectura participativa en el caso barcelonés (Amorós, 1999) 
exigía generar alianzas público-privadas dirigidas a "salvar la situación", dando lugar a un modelo de governance asimétricamente configurado en términos de relaciones de poder (Swyngedouw, 2005) que restringía el espacio de actividad asociativa sólo a la ejecución de servicios culturales de proximidad.

En este escenario, proliferaron mecanismos estables (Consejos, Comisiones, fórmulas de governace en diversos niveles de co-gestión y tipo de consorciarización) y procedimientos temporales limitados (Procesos participativos, Foros, Audiencia Pública, Iniciativa y Consulta Ciudadana) tanto territoriales como sectoriales para los que se previeron funciones de información, consulta y deliberación, en ningún caso vinculantes y por tanto muy proclives a la baja intensidad participativa.

Además, la imposibilidad de la elección directa de los Concejales de Distrito (Bonet-Martí, 2012) y desde los '90 la caída progresiva del presupuesto cultural asignado a los Distritos dentro del presupuesto cultural global del Ayuntamiento ${ }^{8}$ junto al giro gerencialista de la administración local (Brugué y Gomà, 1998) influyeron en la desactivación de la dimensión política de la descentralización.

Por último, el gasto principal de los Distritos correspondía a la creación y mantenimiento de infraestructuras sin embargo, la falta de planificación sobre la función y valor de los equipamientos de proximidad allí situados ${ }^{9}$ puso en cuestión estas actuaciones por su magnitud en el presupuesto municipal frente a su escaso retorno sociocultural en el territorio (Sánchez Belando, 2010).

Las entrevistas a miembros de las asociaciones de vecinos y a técnicos del Ayuntamiento dejan ver otros factores implicados en el diseño de unos mecanismos de baja intensidad democrática y alta intensidad técnico-procedimental en el marco institucional, (1) el temor a la gestión del conflicto, a los imprevistos y a la pérdida de control sobre el proceso, (2) el predominio de una visión elitista de la práctica política por parte del gobierno local (3) Con esto, la desconfianza de técnicos y políticos del Ayuntamiento sobre la capacidad de la ciudadanía y las asociaciones para ejercer la participación, (4) La visión de la participación como un proceso ralentizador y contradictorio con los principios de eficiencia administrativa.

Otro factor destacado en las entrevistas ${ }^{10}$ es el debilitamiento del movimiento vecinal como agente de presión sobre los asuntos públicos durante la constitución del

${ }^{8}$ En 1990, el gasto cultural de los Distritos dentro del Gasto Cultural Global, representa el 5,7\%, en 1993 el 4.2\%, en 1998 el 4,4\%, en 2001 hay una pequeño incremento al 5,5\% para ir a una tendencia a la baja hasta 2010 año en el que ese gasto es del 3,5\%.

${ }^{9}$ Una excepción en este sentido ha sido el Plan de Bibliotecas de la Diputación de Barcelona, referente como modelo de éxito en términos de valor público e impacto socio-territorial.

${ }^{10}$ Entrevistas a Andrés Naya, Lluis Rabell y Marc Andreu (FAVB) y a Eduard Miralles (Presidente de Interarts). 
primer gobierno democrático en el que se produjo un trasvase ${ }^{11}$ de miembros clave del movimiento al gobierno municipal. Esto cambiaría la correlación de fuerzas entre vecinos y gobierno, por un lado a causa de la pérdida de sus principales cuadros políticos que se integraban al gobierno, pero también por una lectura extendida desde los partidos de izquierda (PSUC-PSC) que interpretaba que el papel asumido por el movimiento vecinal hasta ese momento estaba acabado y que ahora había que dejar que ese nuevo gobierno elegido y nutrido por el propio movimiento actuara. Esto impulsó un proceso de distensión de los vecinos frente al gobierno local; que ha sido interpretado como un mecanismo de desmovilización y aplacamiento (Castells, 1986).

Estos factores se inscriben por tanto, en hechos históricos y políticos del contexto de la transición a la democracia y la formación del primer gobierno local democrático, como en la reestructuración estatal que impuso el giro emprendedor del gobierno local, una situación común a todas las ciudades en transición del modelo de acumulación industrial al de una economía de servicios (Harvey, 1989).

\section{La participación social en el ámbito cultural}

Hablar de participación en el ámbito cultural, genera cierta confusión, ya que de manera generalizada ésta se relaciona con las diferentes teorías de los consumos culturales (Bourdieu, 1988; Van Eijck, 2000; Peterson y Kern, 1996). Esta forma de participación, que se concretaría con la obtención de información, dentro del esquema de análisis de escalas de intensidad participativa, clásico en las ciencias políticas, se sitúa como un prerrequisito para participar (Arnstein, 1969).

Cuando hablamos de participación aquí, hacemos referencia al conjunto de prácticas políticas y sociales a través de las cuales la ciudadanía pretende incidir sobre alguna dimensión de aquello considerado de interés público o común, así como en la acción del gobierno (Parés, 2009; Ibarra Güell et al., 2002; Pateman, 1970; Michels y Graaf, 2010). Desde el punto de vista empírico, en el ámbito cultural, pueden considerarse desde esta perspectiva las políticas socio-culturales de proximidad, ya que la política

11 Jordi Borja, uno de los principales impulsores del modelo de participación barcelonés, había sido parte del trasvase de la militancia vecinal a la estructura del primer gobierno democrático, esto dotaba de legitimidad y confianza a la naciente democracia local a la vista del movimiento de vecinos, y daba cierta garantía de que muchas de las demandas en términos de participación fueran incluidas en el modelo. En el segundo mandato democrático (1983-1987) bajo la alcaldía de Pasquall Maragall, Borja se convierte en Teniente de Alcalde del Área de Descentralización, desde donde dirige el proceso y concreta las primeras normas de participación ciudadana de 1986 (Direcció de Serveis d'Organització i Serveis GeneralsAjuntament de Barcelona, 1985). 
cultural se ha construido como un espacio administrativo poco permeable a la participación de la sociedad civil ${ }^{12}$.

En este terreno lo que se ha promovido es la participación de los profesionales, empresas y organizaciones culturales como una herramienta para la reestructuración y potenciación a partir de la colaboración público-privada (Mascarell, 1995) de un sector que era considerado clave en el giro emprendedor de las ciudades europeas (Harvey, 1989; Degen y García, 2012; Novy y Colomb, 2013; Bianchini, 1993; García, 2004a). Podría decirse que en el ámbito cultural, la participación está condicionada por un lado, por una concepción restringida y elitista de la cultura y por otro, por una noción difusa y tendiente a la instrumentalización que la concibe como un recurso para la resolución de problemáticas sociales (Belfiore, 2002; Connolly, 2011) y para la búsqueda de estrategias de crecimiento en el marco de la economía cultural (Scott, 2010).

En el caso barcelonés, desde el contexto pre-olímpico, la cultura era percibida como un recurso central en la estrategia de regeneración urbana y proyección internacional necesarias para impulsar el modelo de economía de servicios por el que apostaba la ciudad (Rodríguez Morató, 2008; Degen y García, 2012).

Los Planes Estratégicos de 1999 y 2006 y la creación en 2010 del Consejo de Cultura de Barcelona ${ }^{13}$ fueron concebidos, entonces, con estos objetivos de ordenación del sector y deben entenderse como parte de la reestructuración de signo gerencial que había emprendido el Ayuntamiento en 1991 dentro de la cual, en 1995, se crea el Instituto de Cultura de Barcelona (Rodríguez Morató, 2005; Sánchez Belando, Rius y Zarlenga, 2012).

Desde un punto de vista clasificatorio, entre los sistemas de participación institucional presentes en el ámbito cultural, encontramos tipos sectoriales (Planes Estratégicos de Cultura, Mesas sectoriales, Consejo de Cultura, Agenda 21) y territoriales, enfocados a la gestión asociativa de equipamientos (Consejos, comisiones de equipamientos) que se concretan en fórmulas de governance reguladas a través de pliegos de condiciones y contratos de prestación de servicios entre la administración y la entidad gestora.

12 Esta orientación es constatable examinando documentación de la administración cultural local previa a la agencialización y posterior a la creación del ICUB, como el Plan Estratégico 1999 y las actas de las Mesas de Debate del Plan Estratégico 2006. Esto, además ha sido corroborado en las entrevistas a cargos técnicos de la administración cultural, entre ellos el ex Delegado de Cultura Jordi Martí. Por tanto, de facto, y pesar de la inclusión de tres miembros de la sociedad civil en la Junta de Gobierno del ICUB, tal como señalan sus estatutos, la exclusión de la ciudadanía de los mecanismos de participación en cultura es concreta en la práctica.

13 Si bien es cierto que en el caso de la participación sectorial ha habido por parte del gobierno local una preocupación más expresa sobre el tema, también es cierto que los mecanismos de participación son poco efectivos. Primero, porque la dinámica de la que surgen es de arriba hacia abajo, segundo porque no son vinculantes, tercero porque a su interior se establecen relaciones asimétricas entre los actores que participan; un ejemplo de ello es el poder que tienen los sectores culturales más fuertes, como el lobby de las artes escénicas, frente al resto a la hora de orientar la agenda del sector (Rius y Sándhez Belando, 2015) 


\section{Entre la demanda vecinal de Ateneos Populares y la respuesta del gobierno local, los Centros Cívicos}

Si algo caracterizó las reivindicaciones del Movimiento Vecinal durante la transición y los primeros años de la democracia fue su transversalidad respecto de todos los aspectos de la vida cotidiana entre estos, la demanda de espacios de encuentro y sociabilidad, ocio y cultura (Castells, 1986; Domingo i Clota y Bonet i Casas, 1998; Andreu 2010; Borja y de Estudios de Administración Local, 1987). Como en el proceso de descentralización, el Movimiento Vecinal había jugado un papel central en la creación de servicios y equipamientos cívicos y culturales.

En el caso de Barcelona, la creación de Bibliotecas, Centros de producción y difusión artística ${ }^{14}$ y principalmente de Centros Cívicos es el resultado de la presión vecinal sobre el Ayuntamiento (Alabart Vilà et al., 2008), en este sentido, puede decirse que la creación de estos equipamientos resultan de una dinámica bottom-link (Eizaguirre et al., 2012). Las reivindicaciones vecinales se construían sobre dos objetivos ligados a lo cultural: la conservación del patrimonio arquitectónico local y la instalación de Ateneos Populares en estos espacios recuperados.

El Modelo de los Ateneos Populares, Obreros y Libertarios, impulsados entre fines del S. XIX y principios del S. XX como organismos de gestión autónoma del proletariado urbano para la formación y la divulgación científica y artístico-cultural (Aisa, 2000; Aracil et al., 2006; Bonet i Agustí, 1999) permanecía en el imaginario del movimiento vecinal de los años 70 y era lo que inspiraba y movilizaba las demandas vecinales ${ }^{15}$ en el ámbito socio-cultural.

La demanda impulsada entre asociaciones de vecinos y otros actores sociales a través de campañas como "Salvem el Mercat de les Flors" en 1977, "Obrim el Xalet!" contra el derribo del actual Centro Cívico Casa Golferich en 1978 o la Okupación de la antigua fábrica textil La Sedeta que luego se convertirá en las instalaciones del Centro Cívico del mismo nombre (Andreu, M., 2010; Federació d'Associacions de Veïns de Barcelona, 1993) tenían múltiples dimensiones: la defensa de un bien que los vecinos

14 La influencia del movimiento vecinal se dirigió al terreno de las artes escénicas y la música y dentro de éstos ámbitos a la cultura popular tradicional y contemporánea; la creación del Centre Artesà Tradicionàrius y del Ateneo Nou Barris son la consecuencia de esta influencia. La actual sede de Montjuic del Teatre Lliure es también el resultado de la reivindicación de los vecinos del Poble Sec sobre el Mercat de les Flors para convertirlo en un Ateneo Popular cuando el edificio estaba por ser derribado (Alabart Vilà et al., 2008; Andreu, 2010)

15 Entrevistas a Fernando Pindado (participante del proceso del creación del Ateneu Nou Barris y ex técnico del área de participación de la Generalitat de Catalunya, Ferrán Aguiló (miembro del Centro Social del Barrio de Sants, Lluis Rabell, (Presidente de la FAVB) y a Eduard Miralles (Presidente de Interarts y experto en políticas de proximidad, con participación activa en el movimiento vecinal que en los años ' 70 reivindicó la creación de los Centros Cívicos) 
consideraban propio, la provisión de servicios y equipamientos y la gestión asociativa de éstos (Martí-Costa y Martí, 2008). Estas acciones de defensa del patrimonio, dado su carácter flexible, la diversidad de actores implicados (asociaciones de vecinos, miembros del colegio de arquitectos y otros sectores sociales) y movilizados por un objetivo puntual que se agotaba en la resolución del conflicto o representaba la primera fase de éste, encajan en la definición de plataformas de acción (Marti-Costa, M. y Cruz y Gallach, 2010).

Este repertorio de acción (Tilly, 2002), se ha ido replicando desde mediados de los'70 hasta la actualidad, en experiencias como "Salvem Can Ricart" y la campaña del Tic Tac de Can Batlló iniciada en 2009, mostrando una línea de continuidad en las técnicas de presión sobre la acción del gobierno; aunque no en los resultados, cuestión en la cual la inscripción del proceso en un determinado contexto político, económico y social es fuertemente condicionante.

La emergencia de los Centros Cívicos debe entenderse así, como el resultado de la acción e interacción de la sociedad civil y del gobierno local, en el marco de un frágil equilibrio entre los objetivos de bienestar de la agenda socialdemócrata local y las transformaciones y desafíos sociales, políticos y económicos que experimentan las ciudades desde los años ' 80 frente a la crisis de los Estados Nacionales de Bienestar Keynesianos (B. Jessop, 2004: 71-96).

\section{Los Centros Cívicos en el giro emprendedor y creativista de la ciudad}

La revisión de documentación oficial junto a las entrevistas a técnicos municipales, miembros de asociaciones de vecinos y otras organizaciones socio-culturales ${ }^{16}$, dan cuenta de la ambigüedad en la definición de objetivos y funciones de los Centros Cívicos como de su difícil encaje en el modelo emprendedor de base cultural al que se orienta la ciudad desde mediados de los años 80' (Degen y García, 2012; Harvey, 1989; Blakeley, 2005; Capel Sáez, 2010). En este apartado, analizaremos el impacto de esta orientación en los equipamientos a partir de la identificación de momentos significativos para la comprensión de su evolución y cambios como para de las políticas culturales de proximidad.

\subsection{Primera etapa 1979 - 1982}

La provisión de equipamientos de uso socio-cultural en los barrios estaba prevista en el Plan General Metropolitano de 1976; sin embargo, es en el marco de las políticas de

16 Entrevista a la Directora de los Centros Cívicos: La Sedeta, Casa Orlandai, Cotxeres de Sants y Drassanes, al Director de Servicios Personales de Ciutat Vella, la Directora de Servicios Personales del Distrito de Sarriá Sant Gervasi y a la Ex Directora del Servicios Personales del Distrito de Sarría Sant Gervasi. 
regeneración urbana que acompañan la candidatura olímpica de Barcelona, propuesta en 1981 y formalizada en 1982 durante la alcaldía de Pascual Maragall, cuando se materializa la creación de los Centros Cívicos.

El programa de monumentalización de la periferia ${ }^{17}$ y el plan de Centros Cívicos se plantean como parte de esta política de regeneración urbana de base cultural, que reactiva el patrimonio industrial y edificios de interés histórico-arquitectónico, para la instalación de equipamientos culturales (Subirós, 1999) dentro de la tendencia general de las ciudades contemporáneas a situar la cultura en el centro de la dinámica de organización y de desarrollo urbano (Morató, 2008) en lo que se ha denominado el paradigma de la ciudad creativa (Landry y Bianchini, 1995).

Así, la rehabilitación de estas instalaciones como Centros Cívicos respondería desde el principio, no sólo a una necesidad planteada por las organizaciones sociales, sino también, a escala de Distritos, a la orientación culturalista sobre la que se construiría luego el nuevo modelo de desarrollo local que pretendió, equilibrar bajo el denominado Modelo Barcelona, principios de sostenibilidad y las tesis de Florida sobre la ciudad creativa y las clases creativas (Florida et al., 2009; 2002), una combinación llena de contradicciones que ha afectado tanto a la definición como el papel de los Centros Cívicos en la dinámica cultural local (Sánchez Belando, Rius y Zarlenga, 2012).

Desde el punto de vista político, en el contexto pre-electoral de 1979, el discurso del partido Partido Socialista de Cataluña (en adelante PSC) que ganaría las primeras elecciones locales, situaba la participación ciudadana y asociativa como un objetivo prioritario en su programa político.

La política cultural se concebía en este contexto en alineación con el paradigma de la democracia cultural (Zimmer y Toepler, 1996), en el que la implicación de las organizaciones de la sociedad civil eran una parte central del proyecto cultural socialdemócrata. Con este ánimo, el área de cultura se concibe dentro del Área de los Servicios Sociales (PSC, 1979: 63) como un elemento transversal a otras políticas y dirigido al bienestar local, en un sentido cercano a lo que plantea el enfoque de la sostenibilidad (Duxbury y Jeannotte, 2010).

Así, lo que se propone en ese momento es una concepción no estatalista de la cultura, que promueve la inclusión de diversas organizaciones sociales y políticas en su desarrollo e implementación, así como la descentralización cultural como factor de impulso de la "libre expresión de la capacidad creativa popular"(PSC, 1979: 65).

En ese marco, presentan como dos actuaciones fundamentales la creación de Centros Socio-culturales y de Consejos Culturales locales para la participación de asociaciones de vecinos, movimientos urbanos y otras entidades, que frente a la indiferencia del

17 Instalación de esculturas contemporáneas, junto a diferentes elementos de memoria histórica, en los nuevos parques y plazas (Subirós, 1999). 
franquismo con la cultura que no consideraba oficial - en referencia a la alta cultura y los grandes equipamientos- protagonizaban la acción cultural en los barrios (Ibídem: 64).

El papel previsto para las organizaciones sociales en el programa del PSC reconoce la dimensión política de su participación, está visión se mantiene aún durante la primera legislatura del PSC (1979-1982, alcaldía de Narcís Serra) que coincide con la fase de debate sobre el proceso de descentralización, la creación de las primeras Normas Reguladoras de la Participación Ciudadana (a partir de ahora NRPC) (Ajuntament de Barcelona, 1987) y la formulación de la política de equipamientos de proximidad.

\subsection{Segunda etapa $1982-1985$}

El programa de Centros Cívicos se concretó a partir de 1982 durante la alcaldía de Pascual Maragall (PSC) como una política "estrella" en términos de participación, inspirada en modelos existentes en el contexto europeo, como las Maisons de la Culture, los Community Centers y los Centri Civici (Miralles, E. y Saboya M., 2000).

Los principios rectores de estos equipamientos se resumen en: (1) la intervención socio-comunitaria en el marco de la proximidad, (2) la promoción de la participación ciudadana y asociativa, (3) El fomento de la cohesión social, (4) Una visión amplia y transversal de la cultura ligada al paradigma de democracia cultural.

En la nueva configuración administrativa, a causa de la predominante orientación participativa de los equipamientos se opta por inscribirlos en el Área de Descentralización y Participación Ciudadana y no en el Área de Cultura, tras cierto debate ${ }^{18}$ sobre la definición de estos equipamientos en los confluían las áreas de Educación, Cultura, Participación, Servicios Sociales y Salud (Ajuntament de Barcelona, 1984) en el marco de las políticas de servicios personales (Brugué y Gomà, 1998; Leandri, 1989; Sánchez Belando, 2010).

Por un lado el carácter polivalente y la complejidad dada por la multiplicidad de funciones, suponían un reto en términos de su encaje administrativo y modelo de gestión (Sánchez Belando, 2010). Sumado a esto, se planteaba una articulación difícil respecto de las expectativas de capitalidad cultural que tenía Barcelona (Morató, 2008) y de la opción del gobierno local que -aún dentro de los parámetros social-demócratas clásicos- se había volcado de lleno al proyecto de transformación emprendedora de la ciudad (Morató, 2005) en el que las políticas de proximidad eran marginales respecto de las de proyección exterior de la ciudad, implementadas a golpe de grandes eventos y construcción de edificios emblemáticos (Balibrea, 2001; Bianchini, 1993; Capel, 2006; Casellas, Jutgla y Barbera, 2010; García, 2004a), dentro de una tradición que se inicia con las estrategias de boostering clásico (Aronczyk y Powers, 2010) de la Expo-

${ }^{18}$ Entrevista Eduard Miralles, Interarts. 
sición Universal de 1886 (Delgado, 2007), para continuar con las Olimpiadas de 1992 (García, 2012) y el Forum Universal de las Culturas en 2004 (Majoor, 2011).

Por otra parte, un elemento central es que los equipamientos desde su inauguración fueron objeto de controversia "Entre los años ' 70 y principios de los ' 80 , era tanta la movilización social por la demanda de equipamientos culturales y tan habitual la okupación como estrategia de presión sobre el gobierno, que el Ayuntamiento tenía que hacer algo... la respuesta fueron los Centros Cívicos ${ }^{19}$ "Las experiencias de autogestión que resultaban de una okupación, como la del Ateneu Nou Barris, se definían entonces por oposición al modelo de Centros Cívicos, eso era todo lo que no queríamos ser..."20. Frente a los ojos de los vecinos, los Centros Cívicos aparecían como una caricatura o una mala copia de los Ateneos ${ }^{21}$, "como una versión institucionalizada de los Ateneos Populares" 22

La inauguración en 1982 del primer Centro Cívico de la actual red de 51 equipamientos apenas pudo celebrarse a causa de las silbadas y la protesta del movimiento vecinal durante el acto (Miralles, 1993). Los Centros Cívicos no gozaron de una aceptación social generalizada. Eran percibidos como el triunfo de una dinámica top-down, que exigía adecuarse a una participación fuertemente normativizada y renunciar a las expectativas del movimiento vecinal respecto de la gestión comunitaria (con ayuda pública) de espacios socio-culturales pre-existentes, como los Ateneos.

Esto, se alejaba por un lado de la visión inicial del PSC sobre estos asuntos y de los principios de democracia participativa que habían defendido las asociaciones de vecinos durante la primera fase del proceso de descentralización en la que habían estado implicados a través de la Comisión Ciudadana, referida anteriormente.

\subsection{Tercera Etapa $1985-1992$}

En el marco de la implementación de la descentralización, el debate sobre el enfoque político y administrativo de la participación que se había dado entre el gobierno local y las organizaciones sociales entre 1979-1982, cristaliza en Normas, en las que triunfa el enfoque administrativo.

El peso de la dimensión administrativa sobre la política se plasma en la documentación relativa a la Transferencia de Competencias Municipales a los Distritos (Borja, 1985; Ajuntament de Barcelona, 1985) como en la relativa a su organización admi-

19 Entrevista Ferrán Aguiló, Can Batlló.

20 Entrevista Fernando Pindado, antiguo miembro del Ateneu Nou Barris.

21 La descripción no resulta extraña teniendo en cuenta que el diseño de los equipamientos incluye como órgano participativo el Ateneo o Comisión de actividades (Ajuntament de Barcelona, 1984), que podría interpretarse como una concesión simbólica a las demandas del movimiento vecinal.

22 Entrevista Lluis Rabell, FAVB. 
nistrativa y territorial y en las Normas Reguladoras de Participación Ciudadana de 1986 (Ajuntament de Barcelona, 1987) y en su actualización de 2002 (Ajuntament de Barcelona, 2002).

Así, los planteamientos iniciales sobre la participación quedan enmarcados en el nivel discursivo del gobierno local, mientras que en la práctica se produce un giro que se corresponde con la consolidación del enfoque gerencial del nuevo localismo (Brugué y Gomà, 1998; Blakeley, 2005; Geissel, 2009) y la emergencia de un modelo de governance emprendedor (Harvey, 1989; Brenner y Theodore, 2002).

Desde el punto de vista del modelo de gestión y los mecanismos participativos que integran los Centros Cívicos, puede decirse que estos reproducen el triunfo de este carácter técnico-administrativo dentro de un modelo de governance guiado por una visión estratégica y eficientista en relación a las alianzas que establece con actores extra-estatales. En coherencia, se trata de mecanismos de poca eficacia participativa.

El estrechamiento en la práctica de los canales de participación y el equilibrio entre dimensión política y administrativa, se expresan en la definición y funciones del Consejo Rector del Centro Cívico (CCRCC) y del Ateneo o Comisión de actividades, que son los órganos estables de participación que integran.

El CRCC es un órgano de tipo informativo, consultivo, deliberativo y de manera indirecta decisorio, cuyo objetivo es definir las líneas de actuación del equipamiento. Sin embargo, en términos de representatividad, en la práctica, favorece una mayor presencia de miembros del Ayuntamiento que de entidades y usuarios ${ }^{23}$, confirmando una tendencia que ha sido estudiada, la generación de relaciones de desigualdad y asimetrías de poder respecto de quién y cómo se participa (Swyngedouw, 2005; Stoker, 1998). Sumado a esto, este Consejo no es vinculante, cosa que sujeta la concreción de las líneas de actuación a las decisiones a nivel del Distrito o de Municipio (Sánchez Belando, 2010).

El Ateneo o Comisión de actividades es un órgano de mayoría social ${ }^{24}$ (Sánchez Belando, 2010), pero su función es sólo ejecutiva, replicando con esto una forma de delegación de tareas decididas desde arriba, es decir una forma de desconcentración administrativa y no una descentralización del poder político (Amorós, 1995).

Además, las NRPC 1986 y las Normas de Funcionamiento de los Distritos, no registran este órganos, así, su existencia queda condicionada a la voluntad y equilibrios

${ }^{23}$ El CRCC está compuesto por un Regidor del Distrito, dos Consejeros, tres representantes de las áreas vinculadas al Centro Cívico, tres representantes de entidades y tres usuarios (Cfr. Normas de funcionamiento de los Centro Cívicos, Ayuntamiento de Barcelona, 1984). El problema es, tal como señalan los Directores de diversos Centros Cívicos entrevistados, que en los centros de gestión municipal directa, muchas veces la falta de recursos dificulta la dedicación necesaria de sus trabajadores para organizar y difundir las sesiones del Consejo a los usuarios y entidades, como consecuencia, la convocatoria es insuficiente para celebrarlo. Además su actividad en la práctica, cuando es posible, es una pura formalidad

${ }^{24}$ Esta Comisión está compuesta por: Un Consejero del Distrito, un representante de entidades, tres usuarios y tres representantes de actividades. 
de influencia entre los actores públicos, sociales y empresariales implicados en la gestión de cada equipamiento.

En este escenario, las organizaciones sociales, se incorporan como meras proveedoras de servicios públicos, asumiendo un papel ambivalente y contradictorio, que a menudo ha jugado a favor de su despolitización y pérdida de capacidad crítica (Saxby, 1998; Sogge et al., 1998; Zurdo, 2006; Martí-Costa y i Martí, 2008). Asimismo, en esta etapa surgen los Casales de Barrio ${ }^{25}$ con el propósito de ofrecer espacios de encuentro y participación para entidades y vecinos. Sin embargo, si consideramos el denso tejido asociativo local, con 5228 entidades registradas ${ }^{26}$, la red de 23 Casales resulta insuficiente frente a la retirada de la red de 51 Centros Cívicos hacia el terreno artístico-cultural.

\subsection{Cuarta Etapa $1992-1995$}

El proyecto y celebración de los Juegos Olímpicos de 1992, supuso un cambio de perspectiva y un estrechamiento de relaciones entre cultura, urbanismo y estrategia de crecimiento económico de la ciudad, que se encuentra en la base conceptual del llamado Modelo Barcelona, donde la cultura es percibida como un instrumento de cohesión social y de marketing urbano, en el marco de una economía global que incrementa la competitividad entre ciudades por la captación de inversiones y flujos de capital (Degen y García, 2012; García, 2004a; Casellas et al., 2010; Balibrea ,2004).

La aceleración de esta tendencia a partir de 1992, junto a la crisis post-olímpica, pondrá a la vista contradicciones entre los objetivos e impacto de la política cultural en la configuración y usos del espacio urbano (Bianchini, 1993; García, 2004a), la expansión de modelos de gestión público-privados y la externalización de servicios públicos a empresas en un contexto de racionalización del gasto público (Rodríguez Morató, 2005). Estos cambios, afectarán el modelo de gestión de los Centros Cívicos, como su encaje y sostenibilidad dentro del giro emprendedor de base cultural de la ciudad.

En este escenario, una cuestión significativa es el diseño del modelo de voluntariado olímpico, presentado como ejemplar en términos de participación. Sin embargo, de este modelo se ha señalado que contribuyó a consolidar una forma de participación individual y centrada en la prestación de servicios o en la idea de delegación de tareas, representando más que una herramienta de empoderamiento una forma de control e ins-

25 Equipamientos públicos dirigidos al soporte de asociaciones, con instalaciones más reducidas y en menor número que los Centros Cívicos.

26 En Barcelona el 28\% de la población, actualmente 1.620.943 habitantes (Ajuntament de Barcelona, 2013), participa en las 5228 asociaciones registradas (Fuente: Fitxer General d'Entitats Ciutadanes, Ajuntament de Barcelona; Idescat, 2011), en Madrid, se registran 1809 asociaciones en una población de 3.237.937 habitantes (Estadística del Ayuntamiento de Madrid, 2012) y en el caso de Bilbao los datos señalan para 2011, 1204 asociaciones (Fuente: Área de Participación y Distritos del Ayuntamiento de Bilbao) en una población de 353.256 (Ayuntamiento de Bilbao, 2011). 
trumentalización por parte del ayuntamiento sobre las asociaciones de vecinos (Blakeley, 2005; Balibrea, 2004) ${ }^{27}$.

En 1992, se inicia un gradual proceso de externalización ${ }^{28}$ de la gestión de Centros Cívicos, modelo actualmente predominante que supone un nuevo límite a la implicación de las organizaciones sociales, ya que la normativa que regula la adjudicación de la gestión de equipamientos y servicios municipales, no discrimina entre empresas y asociaciones. Esto resulta en la exigencia de unos requisitos técnicos y económicos ${ }^{29}$ que ponen en desventaja al tercer sector y en particular a las asociaciones con menos recursos frente a las empresas (Sánchez Belando, 2010).

La inexistencia de un marco legal específico para la gestión cívica ${ }^{30}$ junto a estas condiciones representa un riesgo de oligopolización en el mercado de servicios de gestión de equipamientos. La distribución de equipamientos externalizados a empresas muestra la plena adhesión de Distritos como Ciutat Vella y el Eixample y la casi total en el resto de Distritos, exceptuando a Sant Andreu, Sants-Mont Juic y Sant Martí, donde hay una mayor presencia de gestión municipal directa, co-gestión (asociativamunicipal) y gestión cívica ${ }^{31}$.

Por otra parte, el achicamiento del gasto público cultural en los Distritos dentro del presupuesto cultural global ${ }^{32}$ abre una etapa de abandono municipal de los equipamientos y de pérdida de eficacia en su función de dinamización territorial en el marco

27 Al respecto, Blakeley recoge en una entrevista de 1997 la percepción de las asociaciones de vecinos sobre la institucionalización de la participación: “así, la política llevada adelante por el Ayuntamiento para las asociaciones de vecinos se puede definir como de "manténganlos tranquilos y felices" (Blakeley, 2005:160)

${ }^{28}$ En 1998, de 38 Centros Cívicos, el 8\% era de gestión empresarial, el 79\% de gestión municipal directa, el 8\% co-gestionados (Ayuntamiento- Asociación) y el 5\% de gestió cívica. En 2009, de 51 centros, el 52\% es de gestión empresarial, el $32 \%$ por el ayuntamiento, el $8 \%$ son de gestión cívica y el $8 \%$ co-gestionados. En 2014, sobre 51 equipamientos, el 63\% corresponde a la gestión empresarial, el 22\% son de gestión directa, el 10\% de gestión cívica, el $4 \%$ cogestionados y el 2\% integran asociaciones y empresas. Datos elaborados a partir del informe Situació actual dels Centres Cívics (febrero 1998), Ajuntament de Barcelona y de información aportada por Direcció de Cultura de Proximitat, Institut de Cultura de Barcelona.

29 Entre estos requisitos, el Distrito de Ciutat Vella, requiere a los adjudicatarios que en un término máximo de 15 dias hábiles desde el siguiente a la publicación, constituya la garantía definitiva a la Tesorería Municipal por un importe de 19. 294, 22 euros. Fuente: Resolución de Adjudicación provisional, Expediente 20105002, Departamento de Administración del Distrito de Ciutat Vella con fecha 25 de mayo 2010.

30 En 2009, nace la Plataforma d'entitats per la Gestió Cívica, que reivindica un marco regulador específico para las organizaciones sociales en los concursos públicos, ya que, si bien, la Carta Municipal y las Normas Reguladoras de Participación Ciudadana contemplan la denominada gestión cívica ésta no está desarrollada ni traspuesta a la regulación de licitaciones y adjudicaciones municipales (Plataforma d'Entitas per la Gestió Cívica, 2012).

31 Fuente: Direcció de Cultura de Proximitat, Institut de Cultura de Barcelona.

32 Cfr. Nota al pie $n^{\circ} 8$. 
de un proyecto de ciudad que continúa potenciando su perfil for export (Borja, 2010) algo apreciable en el balance y definición de líneas de actuación del I Plan Estratégico Económico y Social Barcelona 2000 (Ajuntament de Barcelona, 1990) como en el Programa de Acción Municipal 1992-1995 (Ajuntament de Barcelona, n.d.)

La externalización se aplicó en un escenario de debate y resistencia por parte del mundo asociativo (Miralles, 1993), que intensificó el divorcio entre organizaciones sociales y administración, en el marco mayor de de los primeros signos de la ruptura del consenso ciudadano sobre el Modelo de Ciudad (Borja, 2010; Capel, 2006; Capel Sáez, 2010).

\subsection{Quinta Etapa 1995 - 2004}

A partir de 1995, con la gestión del nuevo responsable de cultura, Ferrán Mascarell la administración cultural y los objetivos de la política cultural se reconfiguran por completo. Retomando el impulso por la capitalidad cultural de la ciudad iniciado por el anterior regidor de cultura, Oriol Bohigas (Bohigas y Barcelona, 1993), Mascarell formaliza el agotamiento de las políticas culturales socialdemócratas para redefinir la política cultural bajo el signo emprendedor de la ciudad (Rius y Sánchez Belando, 2015). Una clara expresión de esta nueva orientación es la creación en 1995 de una Agencia de Cultura (Institut de Cultura de Barcelona), la introducción de la planificación estratégica y la governace como una fórmula generalizada de gestión, esto en el contexto de un giro neoliberalizador y de fuerte influencia y contradicciones del paradigma de las ciudades creativas (Degen y García, 2012; Novy y Colomb, 2013; Martí-Costa y Miquel, 2011)

El primer Plan Estratégico Cultural (1999) toca de manera tangencial la problemática de los Centros Cívicos y a diferencia de lo que ocurre con el Plan de Bibliotecas, los Centros Cívicos no tienen cabida en la planificación cultural, como señalan las entrevistadas, durante este periodo "Nadie quería hablar de los Centros Cívicos", que eran "tierra de nadie" 33

\subsection{Sexta Etapa 2004- 2010}

En el contexto de un nuevo mega-evento, el Fórum Universal de las Culturas 2004, la instrumentalización social (como recurso de cohesión social) y económica (como recurso de visibilidad internacional de la ciudad) de la cultura, llega a su máxima expresión, poniendo sobre la mesa las fuertes contradicciones sobre las que se recostaba el Modelo de ciudad y la orientación de la política cultural (Sánchez Belando, Rius y Zarlenga, 2012; Rius y Sánchez Belando, 2015).

\footnotetext{
${ }^{33}$ Entrevistas Directora del Centro Cívico de La Sedeta y Director del Centro Cívico Cotxeres de Sants.
} 
Durante la Alcaldía de Jordi Hereu (2006-2011, PSC), la ciudad se embarca en un nuevo proyecto de crecimiento a golpe de actuaciones de alto impacto en cuanto a creación de imagen y visibilidad global (García, 2004b) haciendo extensivo el discurso de la creatividad a los Centros Cívicos a través de las líneas estratégicas del Programa de Acción Municipal 2004-2007 (Ajuntament de Barcelona n.d.) y del Plan Estratégico de Cultura 2006 (ICUB, 2006), en los que se refuerzan dos objetivos clave para la adaptación culturalista de los equipamientos, ya sugeridos en el periodo anterior (1) la especialización en un sector cultural (2); la compatibilización entre cultura de proximidad y de ciudad. Con esto se produce, cierto acercamiento entre el ICUB y los equipamientos, al mismo tiempo que los objetivos culturales de proximidad se vuelven más difusos.

\subsection{Séptima Etapa 2010- actualidad}

En gran medida como parte de un cuestionamiento generalizado sobre el papel de la cultura en el modelo de ciudad (Degen y García, 2012; Balibrea, 2001; Majoor, 2011; Martí-Costa y Miquel, 2011; Novy y Colomb, 2013; Kagan y Hahn, 2011) y en parte como resultado de los retos que plantea la crisis desde 2008 se abre una nueva etapa en la que las organizaciones de la sociedad civil retoman debates abandonados en la década del ' 80 , al mismo tiempo que el Ayuntamiento enfatiza el giro creativista de los equipamientos vinculándolos al ICUB en 2012 (Ajuntament de Barcelona y Institut de Cultura de Barcelona, 2012).

El debate e impulso de la gestión comunitaria vuelve a situarse en la agenda de las organizaciones sociales, que identifican la crisis como una oportunidad para concretarlo. Para esto, en 2009 se crea la Plataforma de entidades por la gestión cívica ${ }^{34}$ compuesta por los directores de centros co-gestionados y que tiene como objetivo la modificación del actual marco legal que rige la adjudicación de la gestión de equipamientos con vistas a mejorar las condiciones para las asociaciones en los concursos públicos.

Por su lado, el Ayuntamiento, también retoma algunos proyectos antiguos, como la concreción del trabajo en red de los equipamientos que siempre estuvo planteada pero nunca se implementó, con el objetivo de disminuir gastos de programación a través de la circulación de producciones por la red y la colaboración en cuestiones técnicas ${ }^{35}$.

Paralelamente, se impulsa en 2010 un Plan Director de Centros Cívicos que pretende acompañar la reconversión artístico-culturalista de los equipamientos, en este marco se lanzan además una serie de campañas para proyectar los centros al ámbito de ciudad a través de exposiciones y programación de eventos en puntos centrales como Plaza Universidad y Rambla Catalunya, como "Saltem les Muralles" y "Tornem a saltar les

${ }^{34}$ Cfr. Nota al pie $\mathrm{n}^{\mathrm{o}} 27$.

35 Entrevista Tony Puig, ex técnico de la desaparecida Gerencia de Educación Cultura y Bienestar de la que dependían los Centros Cívicos hasta 2011. 
Muralles" realizadas entre 2009 y 2010 dentro del Programa Barrios Creativos (Ajuntament de Barcelona y Gerencia de Educació, 2009)

En 2011, con el triunfo electoral de Xavier Trias (Convergencia i Unió) se produce un giro determinante en la instrumentalización y visión economicista de la cultura y la creatividad, que se expresa en Plan Estratégico Metropolitano 2020 (Asociación Plan Estratégico Metropolitano de Barcelona, 2010) a través de objetivos de liderazgo global de la ciudad en cuanto a creatividad, innovación y conocimiento, líneas estratégicas para promover la atracción de talento innovador, en un claro guiño a los planteamientos de Florida (Florida, 2002). En este marco se reconfiguran las Direcciones del ICUB, donde se crean nuevas direcciones como la de Creatividad e Innovación y la de Cultura de Proximidad, en la que por primera vez se incluye a los Centros Cívicos.

\section{Conclusiones}

El objetivo de este artículo ha sido responder a dos interrogantes: ¿Cuál es el impacto del paradigma emprendedor de base cultural por el que ha optado la ciudad de Barcelona sobre las políticas culturales de proximidad? y ¿Cómo afecta este impacto en la dimensión participativa y democrática atribuida a estas políticas?

Respecto de las hipótesis planteadas, hemos podido constatar que la implementación de la descentralización, en el contexto del giro emprendedor de la ciudad (Harvey, 1989) se orientó a favorecer un mayor peso de la dimensión administrativa sobre la política de ese proceso, cosa que propició el diseño de unos mecanismos de participación ciudadana de baja intensidad y una visión instrumental del papel de los actores sociales incorporados a las nuevas alianzas estratégicas público-privadas que promovía el Ayuntamiento.

Esto, planteó un desencuentro en las relaciones entre las organizaciones sociales y el gobierno local, ya que las expectativas de una participación de mayor intensidad - en la toma de decisiones o en la gestión comunitaria de equipamientos socio-culturales- se vieron frustradas, $\mathrm{y}$, aquellas organizaciones sociales que se transformaran en aliados estratégicos de la administración lo harían restringiendo su actividad a la provisión de servicios, en un marco de desconcentración administrativa.

Por otra parte, la cultura, a partir del proyecto olímpico, se había convertido en un recurso estratégico para el marketing urbano. El conjunto de actuaciones que posicionarían a Barcelona en el mercado global de ciudades, se movilizaba en base a grandes eventos, construcción de edificios emblemáticos, de arquitectura de autor y no de equipamientos de barrio.

Como se ha señalado, las presiones por la competitividad internacional desafiaban la sostenibilidad del llamado Modelo Barcelona, mientras los actores sociales locales intentaban asegurar la justicia social en el contexto local (Degen y García, 2012). 
Puede decirse que, por un lado, la desactivación de la dimensión política de la participación ciudadana a la que hemos hecho referencia, junto a la aceleración del paradigma de la creatividad, transformó a los Centros Cívicos en equipamientos subvalorados y desarticulados tanto del giro creativista como de los objetivos de dinamización territorial y promoción de la participación para la que fueron concebidos. Esta situación se ha resuelto con la adscripción de los equipamientos al Instituto de Cultura de Barcelona en 2011, que ha apostado por convertirlos en espacios de consumo cultural en detrimento de su dimensión social. En suma, se observa una reducción de los espacios de titularidad pública para estos fines, ya que los Casales de Barrio, resultan escasos para suplir los objetivos iniciales de los Centros Cívicos.

En este escenario de apuesta por un modelo de ciudad for export, las políticas dirigidas al bienestar, como las de proximidad y en ese marco el Programa de Centros Cívicos, se ven desplazadas, ilustrando algunos de los dilemas señalados por Bianchini (1993) hace más de dos décadas: la tensión entre proyección internacional y grandes eventos frente a actuaciones dirigidas al desarrollo y el bienestar local, entendidas como parte de un proceso de profundización democrática.

\section{Bibliografía}

Aisa, F. (2000): Una Història de Barcelona:Ateneu Enciclopèdic Popular, 1902-1999, Barcelona, Virus.

Ajuntament de Barcelona (1984): Normas de funcionamiento de los Centros Civicos.

- (1985): Proceso de Descentralización territorial de funciones. Primera etapa, Proyecto de Transferencia no 9, Gestión de los Centros Cívicos.

- (1987): Normes reguladores de l'organització dels Districtes i de la participació ciutadana.

- (1990): Pla Estratègic econòmic i social Barcelona 2000.

- (2002): Normas Reguladoras de la Participación Ciudadana.

- Programa de ACtUaCión Municipal 1992-1995.

- PRograma de Actuación Municipal 2004-2007.

- (2013): Anuari estadístic de la ciutat de Barcelona.

Ajuntament de Barcelona e Institut de Cultura de Barcelona. (2006): Nous accents 2006. Pla estratègic del sector cultural de Barcelona.

Auuntament de Barcelona y Gerencia de Educació. (2009): Barris Creatius.

Ajuntament de Barcelona e Institut de Cultura de Barcelona. (2012): Document de treball per l'elaboració del pla dels centres cívics. 
Alabart Vilà, A., M. Acebal y R. CÁceres (2008): La Barcelona dels Barris Federació d'Associacions de veïns de Barcelona, ed., Barcelona, Mediterrània.

Amorós, M. (1995): Decentralisation and New Governance: A comparison betwen Barcelona and Birmingham, Working Papers Universitat Autònoma de Barcelona, (110).

- (1996): Decentralisation in Barcelona, Local Government Studies, 22(3).

- (1999): La Descentralización en Barcelona y Birmingham. Análisis comparado de los procesos de descentralización política y administrativa en el Ayuntamiento de Barcelona y en el Birmingham City Council, Barcelona, Fund. Pi i Sunyer d'Estudis Autonòmics i Locals.

Andreu, M. (2010): 1970-2010, 40 Anys D’acció Veïnal Federació D’Associacions de Veïns de Barcelona, ed., Barcelona, Mediterrània.

Aracil, R. et al. (2006): Memòria de la transició a Espanya i a Catalunya volum VI $i$ $V I I$ :ensenyament, cultura i justícia, Barcelona, Publicacions i Edicions de la Universitat de Barcelona.

Arnstein, S.R. (1969): A Ladder Of Citizen Participation. - Journal of the American Planning Association, (- 4), p.- 216-224.

Asociación Plan Estratégico Metropolitano de Barcelona (2010): Pla Estratègic Metropolità de Barcelona.

Ayuntamiento de Bilbao (2011): Bilbao en cifras.

BALIBREA, M.P. (2001): Urbanism, culture and the post-industrial city: Challenging the "barcelona model", Journal of Spanish cultural studies, 2(2), pp.187-210.

- (2004): "Barcelona: Del modelo a la marca", en I. E. N. y L. G.-M. J. Carrillo (ed.). Desacuerdos 3. sobre arte, políticas y esfera pública en el estado español, Barcelona, Arteleku — MACBA- UIA., pp. 261-271.

Belfiore, E. (2002): Art as a means of alleviating social exclusion: Does it really work? A critique of instrumental cultural policies and social impact studies in the UK, International Journal of Cultural Policy, 8(1), pp.91-106.

BianchinI, F. (1993): «Remaking European Cities: the role of cultural policies», en F. Bianchini \& M. Parkinson (eds.), Cultural Policy and Urban Regeneration: the West European Experience. Cultural Policy and Urban Regeneration: The West European Experience, Manchester, Manchester University Press, pp. 1-20.

BIER, A.G. (1979): «El desarrollo de las Asociaciones de Vecinos en España», Papers, Revista de Sociologia, (11).

Blakeley, G. (2005): "Local Governance and Local Democracy: The Barcelona Model", Local Government Studies, 31(2), pp.149-165.

Bohigas, O. y Barcelona (1993): Gràcies i desgràcies culturals de Barcelona, Barcelona, Ajuntament de Barcelona. Àrea de Cultura. 
Bonet i Agustí, L. (1999): Evolución y retos de la política cultural en España, Tablero, Revista del Convenio Andrés Bello, (61), pp.89-99.

BorJa, J. (1985): “Descentralització. Una qüestió de mètode”, Autonomies, Revista catalana de derecho público, (2-3), pp.21-46.

BorJa, J. (2010): Llums i ombres de l'urbanisme de Barcelona, Barcelona, Empúries.

BorJA, J. (1977): "Popular movements and urban alternatives in post-Franco Spain", International Journal of Urban and Regional Research, 1(1-4).

Borja, J. e Instituto de Estudios de Administración Local (1987): Descentralización y participación ciudadana, Madrid, Instituto de Estudios de Administración Local.

Bourdieu, P. (1988): La Distinción :criterios y bases sociales del gusto, Madrid, Taurus.

BrenNer, N. \& N.TheOdORE (2002): Spaces of neoliberalism :urban restructuring in North America and Western Europe, Malden Mass., Blackwell.

Brugué, J. y Gomà, R. (1998): Gobiernos locales y políticas públicas: bienestar social, promoción económica y territorio, Barcelona, Ariel.

CAPEl, H. (2006): "De nuevo el Modelo Barcelona y el debate sobre el urbanismo barcelonés", Revista bibliográfica de geografía y ciencias sociales (Serie documental de Geo Crítica, XI(629).

Capel SÁez, H. (2010): "Epílogo ¿ En qué ha fallado Barcelona “, Scripta Nova, 90, pp.173-204.

Casellas, A., E. Jutgla, E. y M.P. Barbera (2010): "Creación de imagen, visibilidad y turismo como estrategias de crecimiento ec onómico de la ciudad", Finisterra, XLV(90), pp.153-172.

Castells, M. (1986): La Ciudad y las masas: sociología de los movimientos sociales urbanos, Madrid, Alianza.

Castells, M. E I.R. De Solís (1985): Movimientos sociales urbanos, 8a ed., México, Siglo XXI de España.

Connolly, M.G. (2011): "The "Liverpool model(s)": cultural planning, Liverpool and Capital of Culture 2008", International Journal of Cultural Policy, pp.1-20.

Degen, M. y M. García (2012): «The Transformation of the "Barcelona Model": An Analysis of Culture, Urban Regeneration and Governance», International Journal of Urban and Regional Research, 36(5), pp.1022-1038.

Delgado, M. (2007): La Ciudad mentirosa :fraude y miseria del “modelo Barcelona”, Madrid, Los Libros de la Catarata.

Domingo i Clota, M. y M.R. Bonet i Casas (1998): Barcelona i els moviments socials urbans, Barcelona, Mediterrània. 
Duxbury, N. \& S. Jeannotte (2010): "From the bottom-up: culture in community sustainability planning. Culture and the Making of Worlds", 3rd ESA Sociology of Culture research mid-term Conference, pp. 1-15.

VAn Eusck, K. (2000): «Richard A. Peterson and the culture of consumption», Poetics, 28(23), pp.207-224.

EizaguiRre, S. et al. (2012): «Multilevel Governance and Social Cohesion: Bringing Back Conflict in Citizenship Practices», Urban Studies, 49(9), pp.1999-2016.

Fainstein, S.S. \& C. Hirst (1995): Fainstein, N., en D. et al. Judge, ed. Theories of Urban Politics. London, Sage.

FLORIDA, R.L. (2002): The Rise of the creative class :and how it's transforming work, leisure, community and everyday life, New York, Basic Books.

Florida, R.L., R. T. i Balcells y A.G. ARnau (2009): Les Ciutats creatives: com l'economia està convertint la tria de l'indret on viure en la decisió més important de la teva vida, Barcelona, Pòrtic.

GARCíA, B. (2004a): «Cultural policy and urban regeneration in Western European cities: lessons from experience, prospects for the future», Local Economy, 19(4), pp.312-326.

García, B. (2012): The Olimpic Games and Cultural Policy, New York - London, Routledge.

GARCÍA, B. (2004b): «Urban regeneration, arts programming and major events: Glasgow 1990, Sydney 2000 and Barcelona 2004», The international journal of cultural policy, 10(1), pp.103-118.

Geissel, B. (2009): «Participatory Governance: Hope or Danger for Democracy? A Case Study of Local Agenda 21», Local Government Studies, 35(4), pp.401-414.

GonzÁLEZ, S. (2011): "Bilbao and Barcelona "in Motion". How Urban Regeneration "Models" Travel and Mutate in the Global Flows of Policy Tourism", Urban Studies, 48(7), pp.1397-1418.

Harvey, D. (1989): «From Managerialism to Entrepreneurialism: The Transformation in Urban Governance in Late Capitalism», Geografiska Annaler.Series B, Human Geography, 71(1, The Roots of Geographical Change: 1973 to the Present), pp. 3-17.

- (2001): Spaces of Capital: towars a critical geography, New York, Routledge.

- (2013): Ciudades Rebeldes. Del derecho a la ciudad a la revolución urbana, Madrid, Akal.

Ibarra Güell, P., S. Martí i Puig y R. Gomà (2002 ): Creadores de democracia radical: movimientos sociales y redes de políticas públicas, Barcelona, Icària.

Jessop, B. (2004): Multi-level Governance and Multi-level Metagovernance. In I. Bache \& M. Flinders, eds. Multi-level Governance, Oxford, Oxford University Press, pp. 49-74.

Jessop, B. \& N. Sum (2000): «An Entrepreneurial City in Action : Hong Kong 's Emerging Strategies in and for ( Inter) Urban Competition», 37(12), pp.2287-2313. 
Jessop, R. y J.C. Monedero (2008): El Futuro del estado capitalista, Madrid, Los Libros de la Catarata.

Kagan, S. \& J. Hahn (2011): “Creative cities and (Un) sustainability: From Creative Class to SustainableCreative Cities", Culture and Local Governance, 3(1-2), pp.11-27.

LANDry, C. \& F. BiAnchini (1995): The Creative city, London, Demos.

LEANDRI, R.G. (1989): Centros cívicos, presente y futuro :cultura y participación, Madrid, Editorial Popular.

Lopez de Aguileta, I. (2000): "Centros Cívicos y sociedad civil: asociacionismo, participación y voluntariado (II)", en J. Perez Gomez (ed.), Centros Cívicos y Servicios de Proximidad. Vitoria - Gasteiz, Xabide, pp. 109-118.

Majoor, S. (2011): "Framing Large-Scale Projects: Barcelona Forum and the Challenge of Balancing Local and Global Needs", Journal of Planning Education and Research, 31(2), pp.143-156.

Martí-Costa, M. y J.B. i Martí (2008): «Los movimientos urbanos: de la identidad a la glocalidad», Scripta Nova, XII(270).

Martí-Costa, M. \& M. I. Pradel (2011): “The knowledge city against urban creativity? Artists' workshops and urban regeneration in Barcelona", European Urban and Regional Studies.

Marti-Costa, M. y H. Cruz y Gallach (2010): “Conflictos urbanísticos y movilizaciones ciudadanas: reflexiones desde Barcelona”, Finisterra, XLV(90), pp.111-132.

Mascarell, F. (1995):Memòria de constitución del instituto de cultura de Barcelona, Barcelona.

Aronczyk M. \& D. Powers (eds.) (2010): Blowing Up the Brand: Critical Perspectives on Promotional Culture, New York, Peter Lang.

Michels, A. \& L. GraAf (2010): «Examining Citizen Participation: Local Participatory Policy Making and Democracy», Local Government Studies, 36(4), pp.477-491.

Miralles, E. (1993): «Dossier, Centres Cívics versus Atenues Populars», en Centres Cívics, què en fem? La veu del Carrer, Federació d'Associacions de Vë̈ns de Barcelona, Barcelona, p. 9.

Miralles, E. y M. Sabolla (2000): “Aproximaciones a la proximidad. Tipologías y trayectorias de los equipamientos en Europa y en España”, Centre d'Estudis i Recursos Culturals de la Diputació de Barcelona.

Montero, J.R., J. Font y M. Torcal (2006): Ciudadanos, Asociaciones y Participación en España., Madrid, CIS.

Morató, R., A. (2008): La emergencia de una capital cultural europea, en M Degen, García, M. y Cavalcanti, L. (eds.), La Metaciudad: Barcelona: transformación de una metrópolis, Rubí, Anthropos, pp. 45-63. 
Morató, R., A. (2005): "La reinvención de la política cultural a escala local: el caso de Barcelona", Sociedade e Estado, 20(2), pp.351-376.

Novy, J. \& C. Colomb (2013): "Struggling for the Right to the (Creative) City in Berlin and Hamburg: New Urban Social Movements, New "Spaces of Hope"? «, International Journal of Urban and Regional Research, 37(5), pp.1816-1838.

PARÉs, M. (2009): Participación y calidad democrática :evaluando las nuevas formas de democracia participativa, Barcelona, Ariel.

Partido Socialista de Catalunya (1979): Programa Eleccions Municipals.

Pateman, C. (1970): Participation and democratic theory, Cambridge, Cambridge University Press.

PeCK, J. (2005): «Struggling with the Creative Class», International Journal of Urban and Regional Research, 29(4), pp.740-770.

Peterson, R.A. \& R.M. Kern (1996): Changing highbrow taste: Fron snob to omnivore, American Sociological Review, 61(5), pp.900-907.

Plataforma D'Entitas per la Gestió Cívica (2012): Document de Presentació de la Plataforma d'Entitats per la Gestió Cívica.

Rius, J., y M.V. SÁnchez Belando (2015): "Modelo Barcelona y política cultural: usos y abusos de la culturaL por parte de un modelo emprendedor de desarrollo local", EURE - Revista Latinoamericana de Estudios Urbano Regionales, 41(122), pp.103-123.

Sánchez Belando, M.V. (2010): La participación cultural en los Centros Cívicos de la ciudad de Barcelona: divergencias y convergencias entre modelos de gestión indirecta. Tesina de Máster, Programa del Máster en Gestión Cultural, Universidad de Barcelona.

Sánchez Belando, M.V, J. Rius y M.I. Zarlenga (2012): “¿Ciudad creativa y ciudad sostenible?: Un análisis crítico del "modelo Barcelona" de políticas culturales", Revista Crítica de Ciências Sociais, (99), pp.31-50.

SAXBY, J. (1998): “¿A quién pertenecen las organizaciones de cooperación no gubernamentales?", en D. Sogge, K. Biekart, y J. Saxby (eds.), Compasión y cálculo: un análisis crítico de la cooperación no gubernamental al desarrollo, Barcelona, Icaria, pp. 65-102.

ScHön, D. \& M. ReIN (1994): Frame reflection: toward the resolution of intractable policy controversies, New York.

Sogge, D., K. Biekart y J. SAXby (1998): Compasión y cálculo :un análisis crítico de la cooperación no gubernamental al desarrollo, Barcelona, Icaria.

Stoker, G. (1998): "Governance as theory: five propositions", UNESCO. Blackwell Publishers., pp.17-28.

SuBIRATS, J. et al. (2008): El Retorn social de les polítiques culturals, Barcelona: Generalitat de Catalunya. Departament de Cultura i Mitjans de Comunicació. 
SuBIRós, P. (1999): Estratègies culturals i renovació urbana, Barcelona, Aula de Barcelona.

Swyngedouw, E. (2005): "Governance Innovation and the Citizen: The Janus Face of Governance-beyond-the-State", Urban Studies, 42(11), pp.1991-2006.

Tilly, C. (2002): «Repertorios de acción contestataria en Gran Bretaña, 1758-1834», en M. Traugott (ed.), Protesta social: repertorios y ciclos de la acción colectiva, Barcelona, Hacer, pp. 17-47.

Zimmer, A. \& S. Toepler (1996): "Cultural Policies and Welfare state: The case of Sweden, Germany and the United States", The journal of arts management, law, and society, 26(3), pp.167-193.

ZuKIN, S. (1989): Loft living :culture and capital in urban change, New Brunswick N.J., Rutgers University Press.

- (1992): «Posmodern urban landscapes: mapping culture and power», en S. Lash y J. Friedman (eds.), Modernity and identity, Oxford, Basil Blackwell, p. 221.

- (1995): The Cultures of cities, Oxford, Blackwell.

Zurdo, A. (2006): «Voluntariado y Estado: Las funciones ambivalentes del Nuevo Voluntariado», Politica y Sociedad, 43(1), pp.169-188. 